\title{
Diosgenin relieves goiter via the inhibition of thyrocyte proliferation in a mouse model of Graves' disease
}

\author{
Hu CAI ${ }^{1,2,3}$, Zhe WANG ${ }^{1}$, Hai-qing ZHANG ${ }^{1,2,4}$, Fu-rong WANG ${ }^{5}$, Chun-xiao $\mathrm{YU}^{1,2,4}$, Feng-xia ZHANG ${ }^{1,2,6}$, Ling GAO ${ }^{1,2,4}$, \\ Jian $\mathrm{ZHANG}^{7, *}$, Jia-jun ZHAO ${ }^{1,2,4, *}$ \\ ${ }^{1}$ Department of Endocrinology, Provincial Hospital affiliated to Shandong University, Ji-nan 250011, China; ${ }^{2}$ Shandong Clinical Medical \\ Center of Endocrinology and Metabolism, Ji-nan 250011, China; ${ }^{3}$ Department of Endocrinology, Taizhou First Peoples' Hospital, \\ Taizhou 318000, China; ${ }^{4}$ Institute of Endocrinology and Metabolism, Shandong Academy of Clinical Medicine, Ji-nan 250011, China; \\ ${ }^{5}$ Department of Pharmacology, Shandong University of Traditional Chinese Medicine, Ji-nan 250011, China; ${ }^{6}$ Department of Neurology, \\ Hospital affiliated to Shandong University of Traditional Chinese Medicine, Ji-nan 250011, China; ${ }^{7}$ Department of Pharmacy, Shandong \\ Provincial Hospital affiliated to Shandong University, Ji-nan 250011, China
}

Aim: To investigate the effects of diosgenin (Dio), a naturally occurring steroid saponin, on goiter formation in a mouse model of Graves' disease (GD) and the underlying mechanisms.

Methods: Female BALB/c mice were injected with adenovirus expressing the A subunit of thyrotropin receptor to induce GD. The mice were treated with Dio $\left(20,100 \mathrm{mg} \cdot \mathrm{kg}^{-1} \cdot \mathrm{d}^{-1}\right.$, ip) for 12 or $24 \mathrm{~d}$. The serum levels of TT4 and TRAb were examined using radioimmunoassay and electrochemiluminescence. The size and morphology of thyroid glands were examined. Thyrocyte proliferation was determined using BrdU incorporation assay. The expression of proliferation-associated proteins IGF-1, NF-KB, cyclin D1, and PCNA in thyroids was analyzed using immunohistochemistry and real-time PCR.

Results: The GD mice showed significantly high serum levels of TRAb and TT4 compared to the normal mice. Treatment of the GD mice with Dio for 24 d dose-dependently reduced the TT4 level and thyroid size, but did not affect the abnormal level of TRAb. Furthermore, Dio treatment dose-dependently reversed the morphological changes and reduced excessive thyrocyte proliferation in thyroids of the GD mice. Dio treatment also dose-dependently reduced the mRNA and protein levels of IGF-1, NF-KB, cyclin D1, and PCNA in thyroids of the GD mice.

Conclusion: Dio relieves goiter in a mouse model of GD through the inhibition of thyrocyte proliferation. The mechanisms involve the suppression of IGF-1, NF-KB, cyclin D1, and PCNA expression.

Keywords: diosgenin; saponin; thyroid gland; goiter; Graves' disease; TRAb; TT4; IGF-1; NF-kB; cyclin D1; PCNA

Acta Pharmacologica Sinica (2014) 35: 65-73; doi: 10.1038/aps.2013.133; published online 18 Nov 2013

\section{Introduction}

Graves' disease (GD) is an autoimmune thyroid disease characterized by diffuse goiter, hyperthyroidism and Graves' ophthalmopathy. Patients with GD produce large amounts of thyrotropin receptor antibodies (TRAb), which bind and activate thyrotropin receptors on thyrocytes, leading to excessive cell proliferation and thyroid hormone secretion, presenting goiter and hyperthyroidism in these patients ${ }^{[1]}$. Over the past two decades, antithyroid drugs, such as carbimazole, methim-

\footnotetext{
* To whom correspondence should be addressed.

E-mail sdjnzhjx@163.com (Jian ZHANG); jjzhao@medmail.com.cn (Jia-jun ZHAO)

Received 2013-07-10 Accepted 2013-08-22
}

azole, and propylthiouracil, have been the preferred mode of therapy, compared with radioactive iodine and thyroid surgery, for the treatment of uncomplicated GD ${ }^{[2]}$. However, there are several disadvantages in antithyroid drug treatment, including a high risk of recurrence, a long course of treatment and the low remission rate of large goiters. For GD patients with large goiters, antithyroid drugs inhibit thyroid hormone synthesis, but these drugs are occasionally powerless to remit goiter, resulting in severe economic burden and psychological pressure on the patients ${ }^{[3]}$. The primary effects of these drugs involve the inhibition of thyroid hormone synthesis through interference with thyroid peroxidase expression, rather than the inhibition of excessive cell proliferation in the thyroid ${ }^{[4]}$. Antiproliferative approaches might be potential therapies for 
GD treatment, particularly for large goiters. Until recently, these drugs had not been implicated in GD treatment.

Diosgenin(Dio), a naturally occurring steroid saponin, is abundantly produced in yam tubers (Dioscorea sp.) and fenugreek seeds (Trigonella sp. ${ }^{[5]}$. Over the past three decades, Dio has primarily been studied for the prevention and/or treatment of various diseases, such as cancer ${ }^{[6]}$, diabetes ${ }^{[7]}$, obesity $^{[8]}$, and dyslipidemia ${ }^{[9]}$. Moalic et al showed that Dio inhibits leukemia and the growth of various cell lines from solid tumors in vitro and in vivo ${ }^{[10-12]}$. The mechanisms of the anticancer activity of Dio involve the modulation of proliferation, apoptotic machinery, and the COX/LOX and cholesterol biosynthetic pathways ${ }^{[6]}$.

Previously, we reported that Dio treatment resulted in thyrocyte $\mathrm{G}_{0} / \mathrm{G}_{1}$ arrest and inhibited the IGF-1-induced proliferation in primary human thyrocytes ${ }^{[13]}$. The ability of Dio to inhibit thyrocyte proliferation could be extrapolated to its prospective chemotherapeutic action against GD. However, these studies have only been performed in vitro because the in vivo environment is much more complicated than using cell lines; thus, we do not know whether the inhibition of Dio on thyrocyte proliferation occurs in vivo. In addition, the effects of Dio on goiter formation in GD remain unclear. Therefore, further in vivo experiments using animal models of GD are needed.

In this study, we applied an adenovirus-mediated mouse model of GD to effectively induce TRAb, goiter and hyperthyroidism ${ }^{[14]}$. We used this model to examine the effects of Dio on goiter formation, and its mechanisms, in the mouse model of GD.

\section{Materials and methods Reagents}

Dr Basil RAPOPORT and Dr Chun-rong CHEN (Cedars Sinai Medical Center, Los Angeles, CA, USA) kindly provided AdTSHR-289 (adenovirus expressing the A subunit of the thyrotropin receptor) and Ad- $\beta$-gal (control adenovirus expressing $\beta$-gal). Dio, Tween 80 and bromodeoxyuridine (BrdU) were purchased from Sigma (St Louis, MO, USA). The total thyroxine (TT4) radioimmunoassay kit was purchased from Jiuding Medical Bioengineering (Tianjin, China), and the TRAb electrochemiluminescence assay kit was obtained from Roche Diagnostics (Basel, Basel-Stadt, Switzerland). For immunohistochemical staining, anti-cyclin D1 antibody was purchased from Boster Biotechnology (Wuhan, China), and all other antibodies were purchased from Santa Cruz Biotechnology (San Francisco, CA, USA). The Streptavidin-peroxidase histostainSP kit was purchased from Zymed Laboratories (South San Francisco, CA, USA). The reagents used for real-time PCR were all purchased from TaKaRa Biotechnology (Tokyo, Japan).

\section{Animals}

Female BALB/c mice (7 weeks old) were obtained from Vital River Laboratory Animal Technology Co, Ltd (Beijing, China). The mice were housed at $23^{\circ} \mathrm{C}$ in $60 \%$ humidity, under a 12 -h light/dark cycle. Food and water were supplied ad libitum.
The care and use of all animals were performed in accordance with the Shandong University Guidelines of the Ethics Committee for Animal Care and Use in research.

\section{Adenovirus preparation}

Ad-TSHR-289 and Ad- $\beta$-gal were propagated in HEK293 cells and purified through $\mathrm{CsCl}$ density-gradient centrifugation. The viral particle concentration was determined by measuring the absorbance at $260 \mathrm{~nm}^{[14]}$.

\section{GD induction and Dio treatment}

All mice were randomly divided into the following groups (16 or 32 animals per group): Norm (Normal), High-dose Dio (100 $\left.\mathrm{mg} \cdot \mathrm{kg}^{-1} \cdot \mathrm{d}^{-1}\right)$, Ad- $\beta$-gal, GD, GD+Low-dose Dio $\left(20 \mathrm{mg} \cdot \mathrm{kg}^{-1} \cdot \mathrm{d}^{-1}\right)$, and GD+High-dose Dio $\left(100 \mathrm{mg} \cdot \mathrm{kg}^{-1} \cdot \mathrm{d}^{-1}\right)$. As shown in Figure 1 , the last four groups were injected intramuscularly with control Ad- $\beta$-gal or Ad-TSHR-289 $\left(10^{10}\right.$ particles in $100 \mu \mathrm{L}$ phosphate buffered saline) three times at 21-d intervals ${ }^{[14]}$. The injection of Ad-TSHR-289 induced GD in mice. At d 21, after the third injection (before Dio treatment), blood samples were collected and tested to verify the GD model. Subsequently, the GD mice received treatment with and without different doses of Dio. The three Dio-treated groups were administered intraperitoneal injections of Dio suspended in $0.1 \mathrm{~mL}$ of $2 \%$ Tween 80 -saline. The mice in the Norm, Ad- $\beta$-gal and GD groups received only $0.1 \mathrm{~mL}$ of $2 \%$ Tween 80 -saline. The animals were euthanized to obtain blood and thyroid gland samples after Dio treatment for 12 or $24 \mathrm{~d}$. The left lobes of the thyroid glands were fixed in $4 \%$ paraformaldehyde for subsequent hematoxylin-eosin (H\&E) staining and immunohistochemistry or in $2.5 \%$ glutaric dialdehyde for electron microscopy. The right lobes were stored in liquid nitrogen until further use for the analysis of mRNA expression.
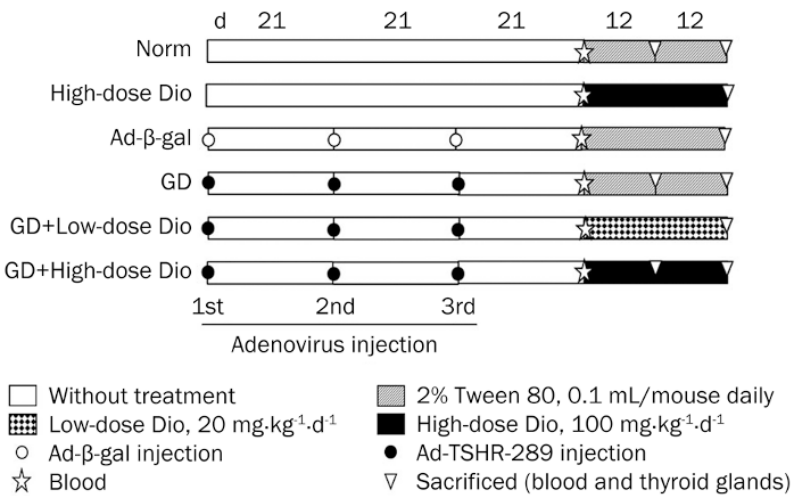

Figure 1. Graves' disease (GD) induction and diosgenin (Dio) treatment procedure. Each icon in the graph represents a different intervention to mice in the experiment. The GD mice were immunized thrice with adenovirus as described in the methods. At $\mathrm{d} 21$ after the third adenovirus injection, different dose Dio was given to the three groups: High-dose Dio, GD+Low-dose Dio and GD+High-dose Dio. Blood samples were taken before Dio treatment. After 12 or $24 \mathrm{~d}$ of Dio treatment, mice were sacrificed, blood and thyroid were collected. 


\section{Serum determinations of TT4 and TRAb}

The TT4 levels in the serum were measured using a radioimmunoassay kit. The serum TRAb concentrations were detected using an electrochemiluminescence assay.

\section{Measurement of thyroid size}

The images of the thyroids were photographed using a digital camera (550D, Canon, Tokyo, Japan). Areas of the thyroid in the images were measured through planimetry using ImagePro Plus software (version 6.0, Media Cybernetics Inc, Silver Spring, MD, USA). The area highlighted in the two-dimensional image represented the size of the thyroid gland.

\section{H\&E staining}

The thyroid tissues were embedded in paraffin, serially dissected into 4- $\mu$ m-thick sections (at least $4-5$ consecutive sections per sample) and subsequently stained with H\&E. The H\&E-stained sections were photographed under a microscope (Leica Microscope Ltd, Wetzlar, Hesse, Germany) and analyzed using Image Pro-Plus software. For each sample, we randomly captured 15 unfolded continuous fields and calculated three parameters: cell density, follicular area and epithelial height.

\section{Transmission electron microscopy}

The thyroid tissues were prepared for electron microscopy using standard procedures, and the ultrastructure was determined through transmission electron microscopy (JEM-100SX, JEOL Ltd, Tokyo, Japan $)^{[15]}$.

\section{Thyroidal BrdU incorporation and detection}

For the BrdU incorporation assay, some mice ( $n=5$ per group) were intraperitoneally injected with BrdU $(50 \mathrm{mg} / \mathrm{kg}$ body weight) twice daily for 3 consecutive days. The mice were euthanized at $2 \mathrm{~h}$ after the last BrdU injection. The thyroid and small intestine were collected. Section preparation, which required special handling, was performed according to Chen et $a l^{[16]}$, and a mouse anti-BrdU antibody (sc-32323, 1:100) was used for staining. For the negative control, the primary antibody was substituted with normal mouse IgG. As one of the most rapidly proliferating tissues in the body, the expression of BrdU in the small intestinal epithelium was strong after BrdU incorporation in vivo ${ }^{[17]}$. Therefore, we used the small intestine with BrdU incorporation as a positive control. For the quantitative analysis of the images, the ratio of the BrdUpositive nuclei to the total number of cells was calculated using Image-Pro Plus software.

\section{Immunohistochemistry}

The sections were prepared as described for H\&E staining. Immunohistochemistry was performed using the following primary antibodies: anti-IGF-1 (sc-9013, 1:400), anti-NF-кB (p65, sc-8008, 1:100), anti-cyclin D1 (BA0770, 1:200) and antiPCNA (sc-7907, 1:200). For the negative control, the primary antibody was substituted with an irrelevant primary antibody of the same isotype. The reactivity of the antibodies was detected using a streptavidin-peroxidase histostain-SP kit. The peroxidase activity was visualized with diaminobenzidine. The sections were counterstained with hematoxylin. The protein expression was quantitatively analyzed using Image-Pro Plus software.

\section{Real-time PCR}

Total RNA was extracted from the mouse thyroid using RNAiso Plus according to the manufacturer's instructions. The cDNA was synthesized using the commercial Takara RT kit. Quantitative real-time PCR assay was performed using SYBR ${ }^{\circledR}$ Premix Ex Taq $^{\mathrm{TM}}$ II in the ABI-PRISM 7700 Sequence Detection System (Foster City, CA, USA). The following primers were used to amplify the mouse genes: IGF-1 (GenBank NM_001111275.1), 5'-TGCTCTTCAGTTCGTGTG-3' and 5'-ACATCTCCAGTCTCCTCAG-3'; cyclin D1 (GenBank NM_007631.2), 5'-GGATGCTGGAGGTCTGTGAG-3' and 5'-CACAACTTCTCGGCAGTCAA-3'; PCNA (GenBank NM_011045.2), 5'-TTTGCACGTATATGCCGAGAC-3' and 5'-GGTGAACAGGCTCATTCATCTCT-3'; and $\beta$-actin (GenBank NM_007393.3), 5'-GGCTGTATTCCCCTCCATCG-3' and 5'-CCAGTTGGTAACAATGCCATGT-3'. The cycling conditions included an initial denaturation step at $95^{\circ} \mathrm{C}$ for $30 \mathrm{~s}$, followed by 40 cycles of $95^{\circ} \mathrm{C}$ for $5 \mathrm{~s}$ and $60^{\circ} \mathrm{C}$ for $30 \mathrm{~s}$. All quantifications were performed using mouse $\beta$-actin as an internal standard. The relative gene expression was analyzed using the $2^{-\Delta \Delta C t}$ method $^{[18]}$.

\section{Statistical analyses}

The data are expressed as the mean \pm standard deviation (SD). All statistics were performed using SPSS 17.0 software (SPSS Inc, Chicago, IL, USA). All variables were examined for normality using the Kolmogorov-Smirnov test. For multiple comparisons, normal data were analyzed using one-way ANOVA followed by the Student-Newman-Keuls test, and non-normal data were analyzed using the Kruskal-Wallis test followed by the Nemenyi test. Statistically significant differences were considered at $P<0.05$.

\section{Results}

Dio decreases the serum level of TT4 not TRAb

As primary features of GD, elevated thyroid hormone and $\mathrm{TRAb}$ levels are typically associated with goiter formation. Hence, we detected serum TT4 and TRAb levels in this study. The levels of TT4 at different time points are shown in Figure 2A. Before the administration of Dio (d 0), the levels of TT4 in both the GD and GD+High-dose Dio groups were higher than those in the Norm group (all $P<0.05$ ), and there was no difference between the GD and GD+High-dose Dio groups. After high-dose Dio treatment for 12 and $24 \mathrm{~d}$, the levels of TT4 were reduced $28 \%(P<0.05)$ and $34 \%(P<0.01)$, respectively, compared with the GD group.

The levels of TRAb and TT4 in all experimental groups after Dio treatment for $24 \mathrm{~d}$ are shown in Figure 2B. The levels of both TRAb and TT4 were increased in the GD group compared with the Norm or Ad- $\beta$-gal groups $(P<0.05$ and $P<0.01$, 

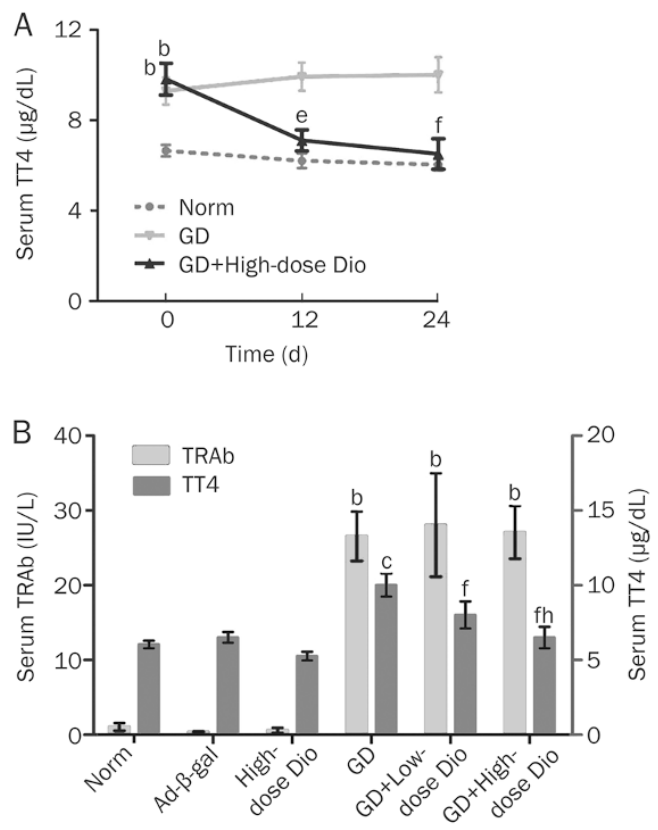

Figure 2. Serum levels of TT4 and TRAb assay. (A) Dynamic TT4 changes during 12- and 24-d Dio treatment. (B) Serum levels of TT4 and TRAb after 24-d Dio treatment. Data are expressed as mean \pm SD $(n=10$ per group). ${ }^{b} P<0.05,{ }^{\mathrm{c}} P<0.01$ vs the Norm group. ${ }^{\mathrm{e}} P<0.05,{ }^{\mathrm{f}} P<0.01$ vs the GD group. ${ }^{\mathrm{h}} P<0.05$ vs the GD+Low-dose Dio group.

respectively). Low- and high-dose Dio treatments for $24 \mathrm{~d}$ reduced the high levels of TT 4 by $20 \%$ and $34 \%$ in the GD mice (both $P<0.01$ ), respectively. A significant dose-dependent difference between mice treated with low and high doses of Dio was detected. Surprisingly, neither low- nor high-dose Dio treatment reduced the levels of TRAb in the GD mice, suggesting that the changes of TT4 after Dio treatment were independent of TRAb.

\section{The effects of Dio on goiter formation in stereology}

Gross inspection (Figure 3A) revealed that the thyroids of the GD group were visually enlarged and dark red in color (goiter) compared with the glands of the Norm or Ad- $\beta$-gal groups. The hypertrophy of thyroids in the GD mice could be suppressed after treatment with low and high doses of Dio. The thyroid size was measured as the computerized planimetry area (Figure $3 \mathrm{~B}$ ). The thyroid areas measured in the GD group were larger than those in the Norm group $(P<0.01)$. There was a slight reduction of the thyroid areas in the GD+Highdose group after Dio treatment for $12 \mathrm{~d}$. However, when Dio treatment was administered for $24 \mathrm{~d}$, the reduced thyroid areas were easier to recognize. Overall, low- and high-dose Dio treatments reduced the thyroid area by $31 \%(P<0.05)$ and $49 \%(P<0.01)$, respectively, compared with the GD group. The significant difference between low- and high-dose treatments reflected the dose-dependent action of Dio.

The effects of Dio on thyroid morphology

H\&E staining revealed that the thyroids observed in the Norm
A
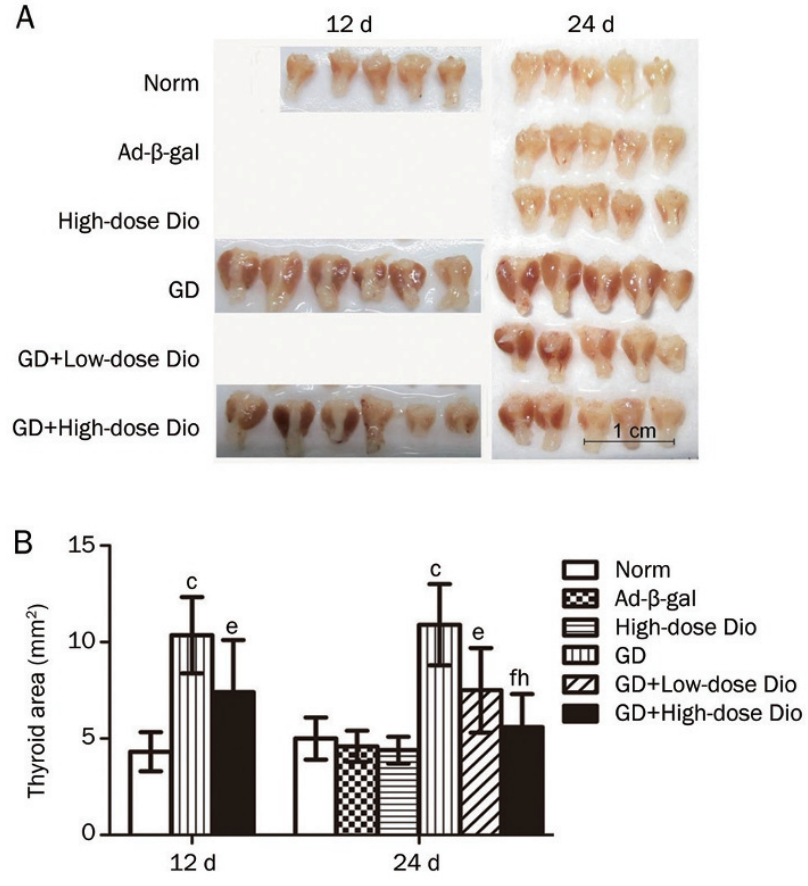

Figure 3. The effects of 12- and 24-d Dio treatment on goiter. (A) External images of thyroid glands. (B) Measurement of thyroid areas by ImagePro Plus software. Data are expressed as mean $\pm S D$ ( $n=5-6$ per group). ${ }^{\mathrm{c}} P<0.01$ vs the Norm group. ${ }^{\mathrm{e}} P<0.05,{ }^{\mathrm{f}} P<0.01$ vs the GD group. ${ }^{\mathrm{h}} P<0.05$ vs the GD+Low-dose Dio group.

group showed normal features under light microscopy (Figure 4A). The thyroids in the GD group were characterized by the predominance of diffuse hypercellularity, hyperplasia and hypertrophy (Figure 4B). These pathological abnormalities were alleviated through Dio treatment, and the effects were more obvious in the high-dose treatment group than those in the low-dose treatment group (Figures $4 \mathrm{C}$ and $4 \mathrm{D}$ ). To
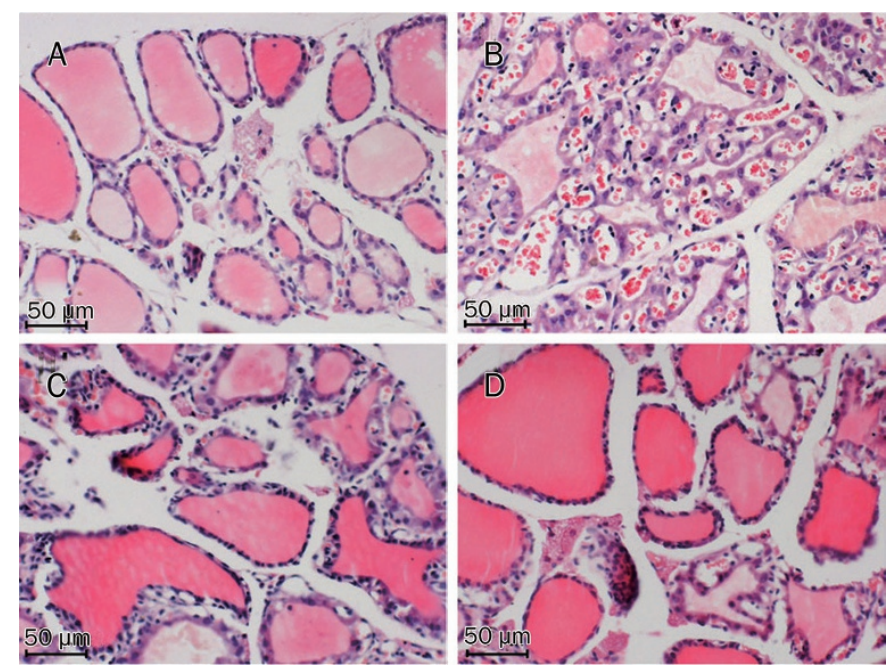

Figure 4. After 24-d Dio treatment, histological changes of thyroid glands stained with H\&E in the Norm (A), GD (B), GD+Low-dose Dio (C), GD+Highdose Dio (D) groups (magnification, $\times 200$ ). 
analyze the histological changes in detail, we measured three parameters, as shown in Table 1. The GD group showed a significant increase in cell density and epithelial height and a significant reduction in the follicular area compared with the Norm group. However, after low- and high-dose Dio treatments for $24 \mathrm{~d}$, these three parameters were significantly restored compared with the GD group. The effects of Dio on cell density and follicular area were dose-dependent (all $P<0.01)$.

The electron micrographs showed that the thyrocytes of the GD group exhibited the characteristics of increased secretion activity (Figures 5A and 5B): these cells showed distorted nuclei, abundant mitochondria, plentiful vesicles of colloid and a rough endoplasmic reticulum with dilated cisternae. These changes were recovered after treatment with high-dose Dio for $24 \mathrm{~d}$ (Figures 5C and 5D).

\section{Dio decreases proliferating thyrocytes labeled by BrdU}

To confirm the effects of Dio on thyrocyte proliferation, we performed a BrdU incorporation assay. As shown in Figures $6 \mathrm{~A}$ and $6 \mathrm{~B}$, the ratio of the BrdU-positive nuclei in the GD group was increased 5.6-fold compared with that in the Norm group $(P<0.01)$. The ratio was significantly reduced after Dio treatment, with a greater reduction in the high dose treatment $(70 \%)$ than in the low dose treatment (29\%).

Dio inhibits the protein expression of IGF-1, NF-KB, cyclin D1, and PCNA in the thyroid

To investigate the mechanism underlying the antiproliferative effects of Dio, we examined the protein expression of IGF-1, NF-kB, cyclin D1, and PCNA in the thyroid using immunohistochemical staining. As shown in Figure 7A, the expression of these proliferation-associated proteins in the GD group was obviously increased compared with the Norm group, and this increase was greatly reduced after a 24-d Dio treatment.

The expression of these proteins was analyzed using ImagePro Plus software. IGF-1-positive cells demonstrated strong, diffuse granular staining in the cytoplasm. The integrated optical density of the positive staining in each image was measured, and the ratio of the density to the area of each image was calculated as the optical density mean, which represented the relative amount of IGF-1 expression ${ }^{[19]}$. Although NF-kB, cyclin D1, and PCNA expression was observed in both the nucleus and cytoplasm, the nuclear overexpression of these proteins has been associated with proliferation ${ }^{[20-22]}$. Thus, we measured the changes in nuclear overexpression of these proteins and calculated the ratio of positive stained nuclei to the total number of cells. The quantitation of these changes is shown in Figure 7B. Compared with the Norm group, the expression of IGF-1, NF-kB, cyclin D1, and PCNA was significantly increased in the GD group. When GD mice were treated with Dio, IGF-1, NF-kB, cyclin D1, and PCNA expression was significantly reduced in the GD+Low-dose Dio group (48\%, $41 \%, 24 \%$, and $29 \%$, respectively) and in the GD+Highdose Dio group $(70 \%, 72 \%, 38 \%$, and $47 \%$, respectively). The Dio-mediated inhibition of the expression of these proliferation-associated proteins was also dose-dependent (all $P<0.05$ ).

\section{Dio inhibits the transcription of IGF-1, cyclin D1, and PCNA in the thyroid}

Furthermore, we performed real-time PCR to characterize the genetic changes. Similar to the changes in protein expression, Dio also reduced mRNA expression after treatment for $24 \mathrm{~d}$ (Figure 8). Compared with the Norm group, the mRNA expression of IGF-1, cyclin D1, and PCNA was significantly increased in the GD group. However, compared with the GD group, the mRNA expression of IGF-1, cyclin D1, and PCNA was significantly reduced in the GD+Low-dose Dio group (46\%, 45\%, and 31\%, respectively) and in the GD+High-dose Dio group $(79 \%, 69 \%$, and $37 \%$, respectively). Significant differences were observed in the mRNA expression of IGF-1 and cyclin D1 between the low- and high-dose Dio treatments.

\section{Discussion}

These results demonstrated that Dio substantially relieved goiter formation in GD mice through the inhibition of thyrocyte proliferation. This study show the potential of Dio as a treatment for GD and may also provide a new therapeutic strategy for GD, involving the prevention of thyrocyte proliferation.

To date, the GD model induced through Ad-TSHR-289 has been widely used in many studies, reflecting the reproducibility of this model ${ }^{[23-25]}$. The changes observed in the GD model in the present study were consistent with those of previous studies $^{[14]}$. The pathogenesis of the mouse GD model was similar to that observed in human GD patients. Mice injected with Ad-TSHR-289 produced high levels of TRAb through

Table 1. Quantification of histological changes in thyroid after 24-d Dio treatment.

\begin{tabular}{lccc}
\hline \multicolumn{1}{c}{ Group } & $\begin{array}{c}\text { Cell density } \\
\left.\text { (cells per mm }{ }^{2}\right)\end{array}$ & $\begin{array}{c}\text { Follicular area } \\
\left(\mu \mathrm{m}^{2}\right)\end{array}$ & $\begin{array}{c}\text { Epithelial height } \\
(\mu \mathrm{m})\end{array}$ \\
\hline Norm & $5890.82 \pm 481.17$ & $2451.55 \pm 305.50$ & $4.28 \pm 0.11$ \\
GD & $9318.95 \pm 962.81^{\mathrm{c}}$ & $1010.00 \pm 375.40^{\mathrm{c}}$ & $10.49 \pm 2.55^{\mathrm{b}}$ \\
GD+Low-dose Dio & $8666.25 \pm 902.71^{f}$ & $1435.75 \pm 258.63^{f}$ & $8.69 \pm 1.64^{f}$ \\
GD+High-dose Dio & $6995.79 \pm 964.16^{\mathrm{fi}}$ & $1823.65 \pm 396.85^{\mathrm{fi}}$ & $6.64 \pm 2.20^{\mathrm{e}}$ \\
\hline
\end{tabular}

Data are expressed as mean \pm SD ( $n=5$ per group). ${ }^{b} P<0.05,{ }^{c} P<0.01$ vs the Norm group. ${ }^{e} P<0.05,{ }^{f} P<0.01$ vs the GD group. ${ }^{i} P<0.01$ vs the GD+Lowdose Dio group. 

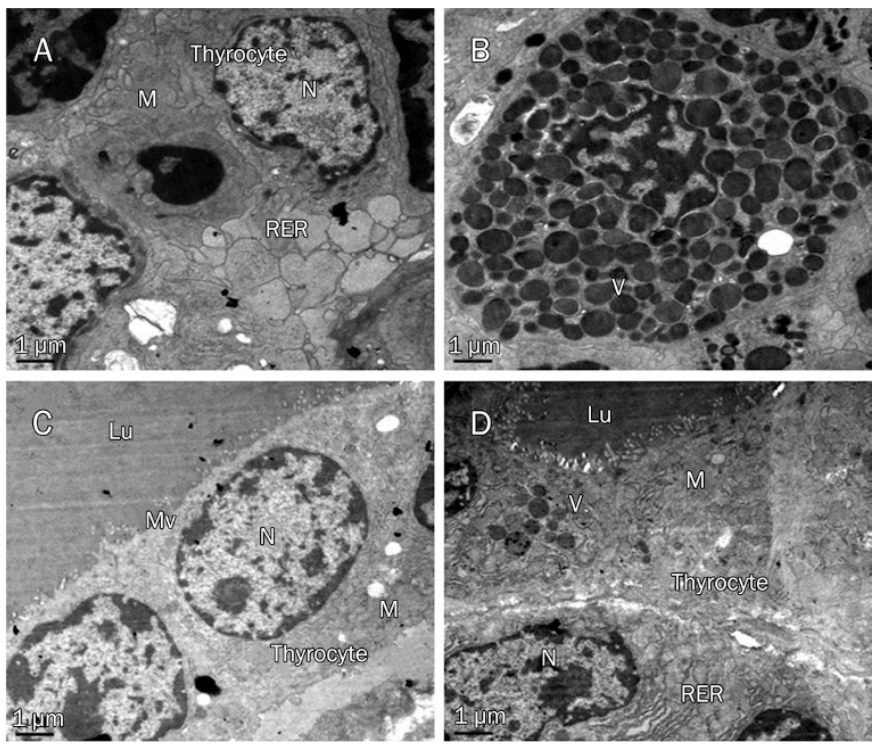

Figure 5. Representative transmission electron micrographs of thyroid glands in the GD (A and $B$ ) and GD+High-dose Dio ( $C$ and $D$ ) groups after 24-d Dio treatment (magnification, $\times 7500$ ). Lu, lumen of the thyroid follicle; M, mitochondrion; Mv, microvilli; N, nucleus; RER, rough endoplasmatic reticulum; $\mathrm{V}$, vesicles of colloid.

the immune response, followed by the induction of excessive thyrocyte proliferation and the eventual onset of GD. The key factors of this disease are TRAb expression and thyrocyte proliferation. The data concerning TT4 expression and thyroid size indicated that Dio was effective in controlling both goiter formation and hyperthyroidism in GD mice. Interestingly, the TT4 expression and thyroid size in normal mice were only slightly affected, even after high-dose Dio treatment, showing statistically insignificant differences. Thus, the effects of Dio selectively affect the proliferating thyroid rather than the normal thyroid, suggesting that Dio might be a safe anti-goiter drug, thereby avoiding hypothyroidism.

In addition to anti-cancer effects, several studies have indicated that Dio modulates immune responses, such as inducing the TNF-a secretion, suppressing IgE production, and upregulating regulatory $\mathrm{T}$-cell production ${ }^{[5,26,27]}$. We speculated that Dio might control goiter through the immunomodulatory inhibition of TRAb expression. Unfortunately, in the present study, Dio treatment did not reduce the high levels of TRAb without impacting the CD4/CD8 T lymphocyte ratio in the spleen (data not shown); however, a reduction in the high level of TT4 expression was observed in GD mice in response to Dio treatment. Consequently, we hypothesized that the target of Dio action might not be TRAb expression but rather thyrocyte proliferation. Therefore, we focused on the changes in the thyroid.

Light and electron microscopy revealed that Dio inhibited hyperplasia, hypertrophy and overactive secretion in the thyroid, which are pathological features of GD. In recent years, many studies have supported the effects of Dio on proliferation, but few studies have shown the effects of this treat-
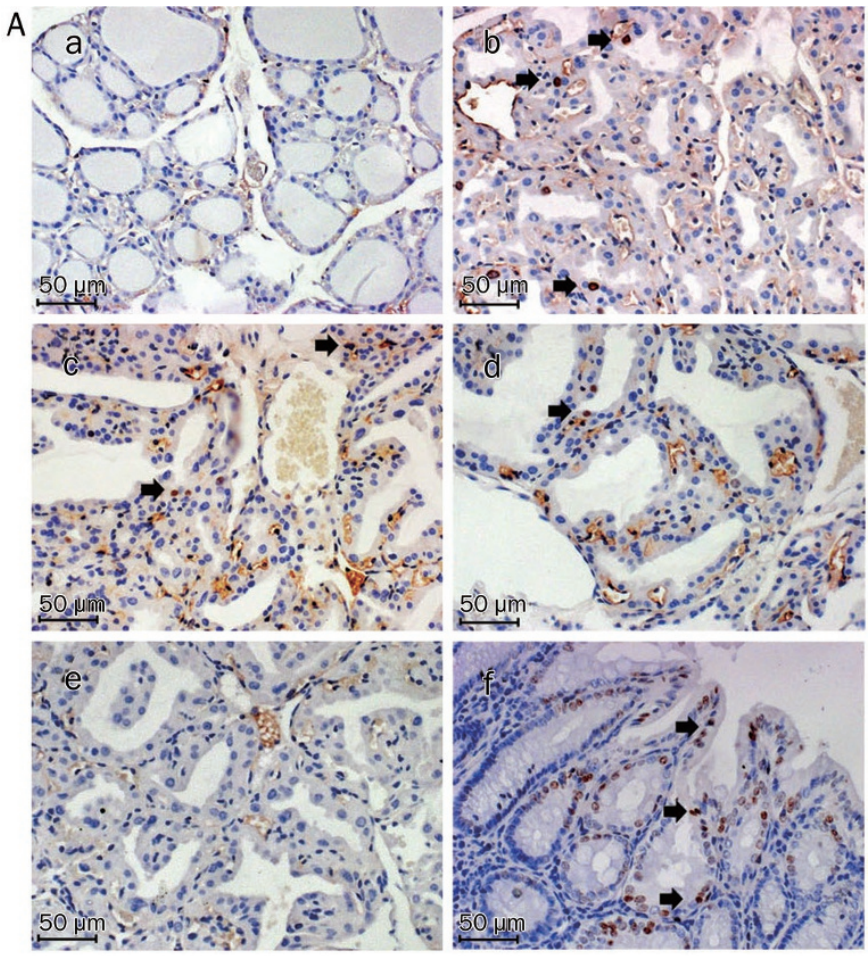

B

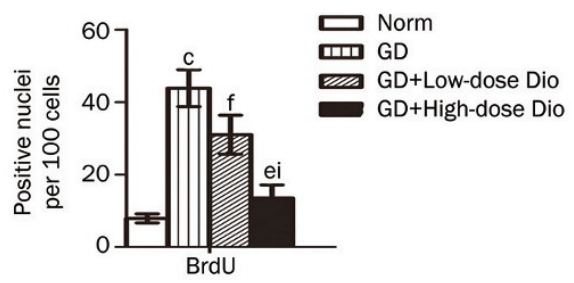

Figure 6. Detection of proliferating thyrocytes labeled by BrdU after 24-d Dio treatment. (A) Representative images of BrdU detection (magnification, $\times 200$ ). After BrdU incorporation in vivo, thyroid samples were obtained from the Norm (a), GD (b), GD+Low-dose Dio (c), GD+High-dose Dio (d) groups. The thyroid and small intestine tissues from the same mouse of (b) were used as the negative control (e) and positive control (f). BrdUpositive nuclei are noted by black arrows. (B) Quantitative analysis of BrdU detection. Data are expressed as mean $\pm S D$ ( $n=5$ per group). ${ }^{\mathrm{c}} P<0.01$ vs the Norm group. ${ }^{\mathrm{e}} P<0.05,{ }^{\mathrm{f}} P<0.01$ vs the GD group. ${ }^{\mathrm{i}} P<0.01$ vs the GD+Low-dose Dio group.

ment on secretion ${ }^{[6]}$. In the present study, the suppression of proliferation was particularly prominent. The action and mechanism of Dio to inhibit thyrocyte proliferation have previously been described in in vitro studies ${ }^{[13]}$. In the present in vivo study, we wanted to characterize the effects of Dio on cell proliferation rather than on thyroxine secretion. However, we cannot rule out the potential effects of Dio on hormone secretion.

Because cell proliferation causes an increase in the number of cells, it is typically characterized by new DNA synthesis. Thus, one of the most accurate and reliable assays for proliferation is to measure DNA synthesis in the cell. BrdU is a synthetic nucleoside that can be incorporated into the newly 
A
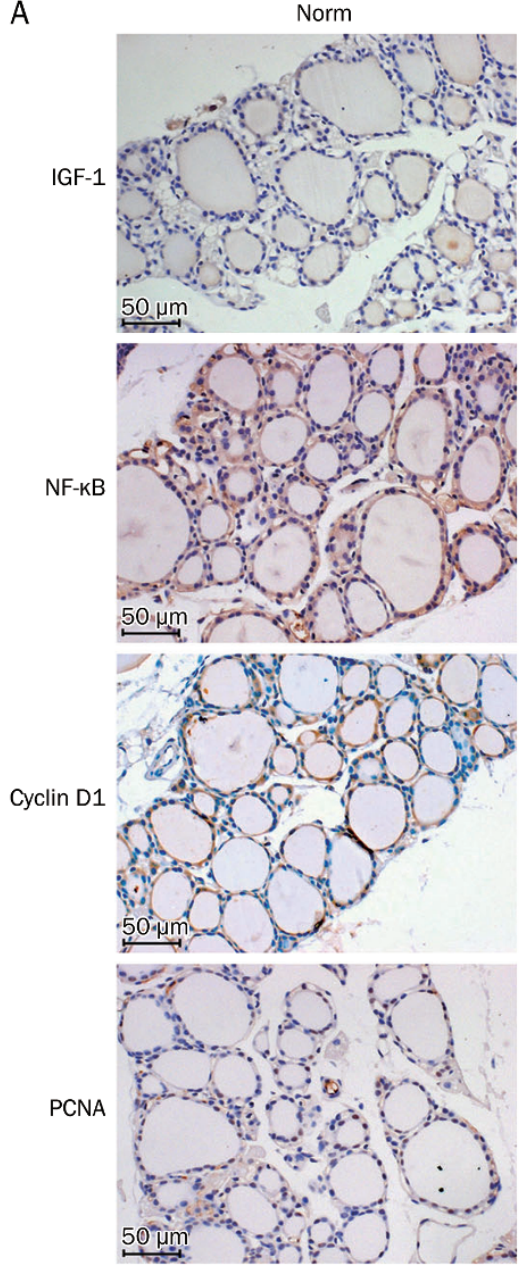

GD
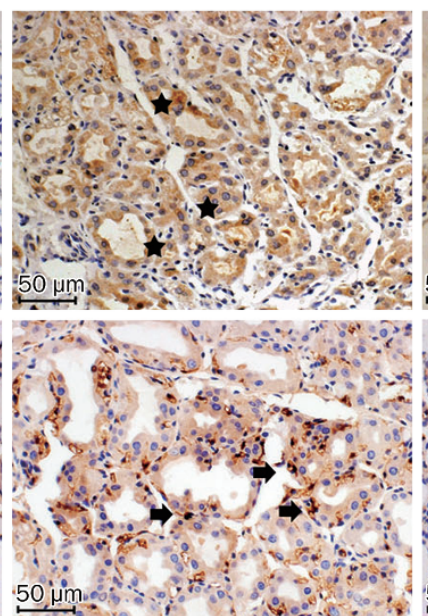
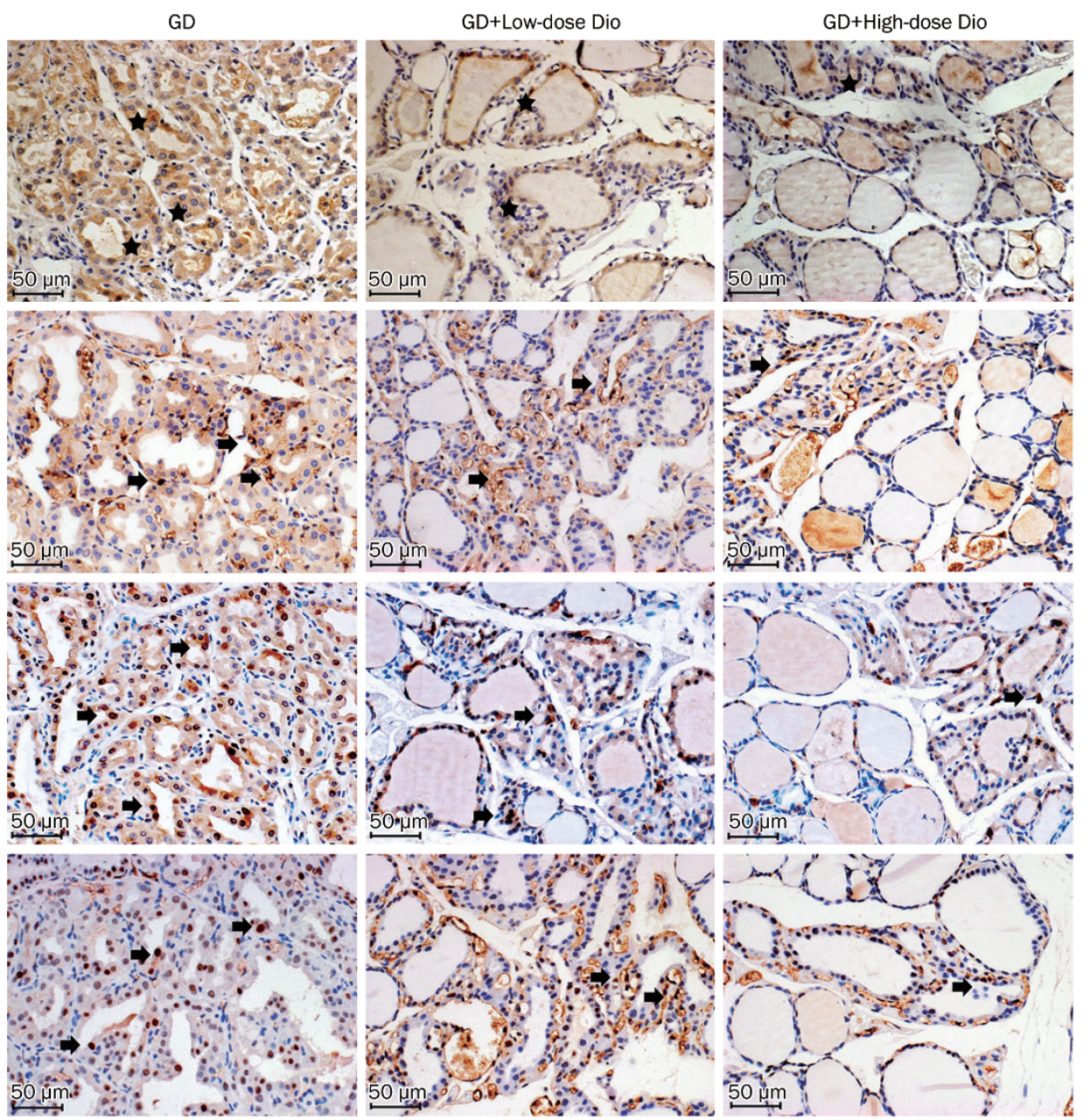

B
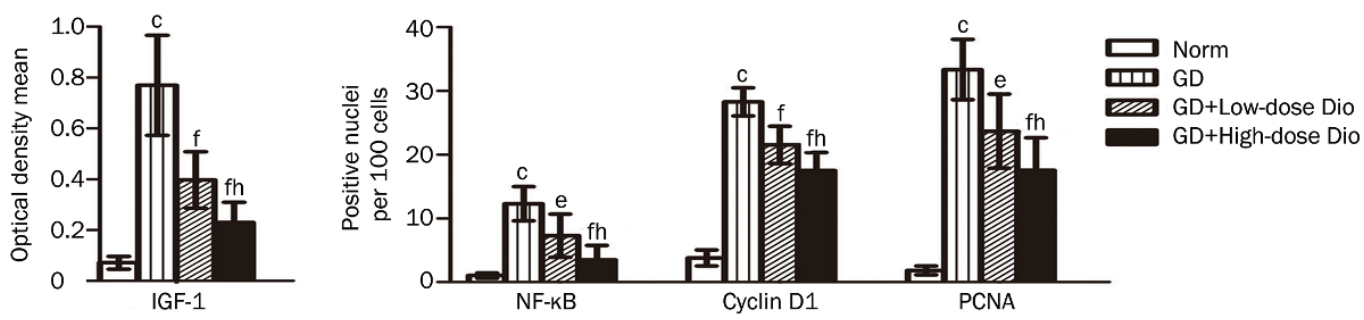

Figure 7. Immunohistochemical staining of IGF-1, NF-KB, cyclin D1, and PCNA in thyroid after 24-d Dio treatment. (A) Representative images of immunohistochemical staining (magnification, $\times 200$ ). Images of the negative control in the assay are not shown because they are similar to that in the BrdU detection above. Positive immunostaining is noted by black stars in the cytoplasm and by black arrows in the nucleus. (B) Quantitative analysis of immunohistochemical staining. Data are expressed as mean $\pm \mathrm{SD}$ ( $n=5$ per group). ${ }^{\mathrm{c}} P<0.01$ vs the Norm group. ${ }^{\mathrm{e}} P<0.05,{ }^{\mathrm{f}} P<0.01$ vs the GD group. ${ }^{\mathrm{h}} P<0.05$ vs the GD+Low-dose Dio group.

synthesized DNA of proliferating cells. BrdU is widely used for the detection of proliferating cells in vivo ${ }^{[28]}$. Consistent with our hypothesis, the results of the BrdU assay showed that proliferating thyrocytes were markedly increased in GD mice, and this proliferation was reduced after Dio treatment. Based on changes in thyrocyte proliferation, we further explored the mechanism underlying the action of Dio. In a previous study, we revealed that Dio inhibits IGF-1-induced thyrocyte proliferation in vitro through a reduction in cyclin D1 expression.
However, we did not examine whether Dio also affected IGF-1 expression ${ }^{[13]}$. IGF-1 is a pleiotropic polypeptide that plays a critical role in cell growth and proliferation. In addition to TRAb, several growth factors are involved in cell proliferation in GD, of which IGF-1 is considered the most important factor. The results of a recent population-based study showed that IGF-1 was intimately associated with goiter formation ${ }^{[29]}$. In addition, clinical studies have indicated that IGF-1 expression was significantly stronger in the thyroid tissue of GD than in 


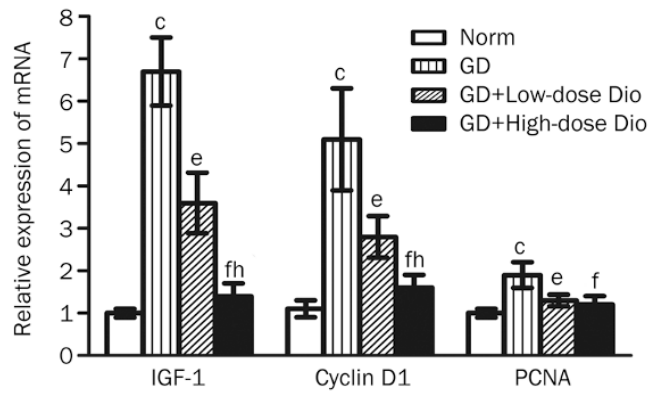

Figure 8. Real-time PCR analysis of IGF-1, cyclin D1, and PCNA in thyroid after 24-d Dio treatment. $\beta$-Actin served as an internal standard. Relative expression was calculated as extent of change with respect to the normal control value. Data are expressed as mean $\pm \operatorname{SD}$ ( $n=5$ per group). ${ }^{c} P<0.01$ vs the Norm group. ${ }^{\mathrm{e}} P<0.05,{ }^{\mathrm{f}} P<0.01$ vs the GD group. ${ }^{\mathrm{h}} P<0.05$ vs the GD+Low-dose Dio group.

normal thyroid tissue ${ }^{[30]}$. Thus, we considered the effect of Dio on IGF-1 in the present study.

In the present study, we observed changes in IGF-1/NF-кB/ cyclin D1/PCNA, which are important molecules in proliferation signaling pathways. It has previously been reported that IGF-1 promotes cell cycle progression through of the upregulation of cyclin D1 expression via the activation of the NF-KB pathway in FRTL thyroid cells ${ }^{[31]}$. NF-KB is a protein complex that plays a key role in regulating cell proliferation and cell survival ${ }^{[32]}$. In the inactive state, NF-kB is located in the cytoplasm, bound to the inhibitory protein ІкBa. As a stimulus, IGF-1 induces the phosphorylation and degradation of IKBa, leading to NF-KB activation. Activated NF-KB enters the nucleus and activates the transcription of target genes ${ }^{[33]}$. Cyclin D1 is likely the most important NF- $\mathrm{kB}$ target gene in the regulation of cell cycle progression ${ }^{[34]}$. Cyclin D1, in association with cyclin-dependent kinase (CDK), phosphorylates the retinoblastoma protein $(\mathrm{RB})$ and promotes the release of the bound E2F transcription factors. These events facilitate the transcription of E2F target genes, which participate in the entry and completion of the $S$ phase ${ }^{[35]}$. One of the E2F target genes is PCNA, a cofactor of DNA polymerase delta that is necessary for DNA synthesis in the S phase ${ }^{[36]}$.

The results obtained in the present study indicate that Dio simultaneously inhibited the overexpression of these proliferation-associated proteins in the thyroid of GD mice, which is consistent with previous in vitro studies ${ }^{[13,37,38]}$. In addition, as a holistic approach in vivo, we also discovered changes upstream and downstream of cyclin D1 in proliferation signaling pathways.

In conclusion, these results show that Dio relieved goiter formation in GD mice. The effect was independent of the TRAb level. The underlying mechanism involved the inhibition of thyrocyte proliferation through the suppression of the gene transcription and protein expression of some proliferationassociated proteins. These findings implicate Dio as a potential new drug candidate for GD treatment. Because Dio has a variety of biological activities, this compound might affect multiple molecules of the proliferation pathway or aspects of GD pathology. Our research has only revealed some of the activities of Dio. Further preclinical and clinical studies are needed to explore the detailed and intensive roles of Dio in GD.

\section{Acknowledgements}

Dr Shu WANG (Ruijin Hospital of Shanghai Jiao Tong University, Shanghai, China) assisted with the delivery of the adenovirus. This work was supported through the National Natural Science Foundation of China (30901461, 81070625 and 81101590), the Natural Science Foundation of Shandong Province (2009CM005 and BS2010YY049) and the Ji-nan Selfrenovation Plan for Colleges and Universities and Scientific Research Institutes (200906012) of China.

\section{Author contribution}

Jia-jun ZHAO, Jian ZHANG, and Ling GAO designed the study; Hu CAI and Zhe WANG performed the research; Haiqing ZHANG and Chun-xiao YU contributed new reagents and analytic tools; Jian ZHANG and Feng-xia ZHANG analyzed the data; and Hu CAI, Ling GAO, and Fu-rong WANG wrote the manuscript.

\section{References}

1 Prabhakar BS, Bahn RS, Smith TJ. Current perspective on the pathogenesis of Graves' disease and ophthalmopathy. Endocr Rev 2003; 24: 802-35.

2 Burch HB, Burman KD, Cooper DS. A 2011 survey of clinical practice patterns in the management of graves' disease. J Clin Endocrinol Metab 2012; 97: 4549-58.

3 Vitti P, Rago T, Chiovato L, Pallini S, Santini F, Fiore E, et al. Clinical features of patients with Graves' disease undergoing remission after antithyroid drug treatment. Thyroid 1997; 7: 369-75.

4 Cooper DS. Antithyroid drugs. N Engl J Med 2005; 352: 905-17.

5 He Z, Tian Y, Zhang X, Bing B, Zhang L, Wang $\mathrm{H}$, et al. Anti-tumour and immunomodulating activities of diosgenin, a naturally occurring steroidal saponin. Nat Prod Res 2012; 26: 2243-6.

6 Raju J, Mehta R. Cancer Chemopreventive and Therapeutic Effects of Diosgenin, a Food Saponin. Nutr Cancer 2009; 61: 27-35.

7 Pari L, Monisha P, Mohamed Jalaludeen A. Beneficial role of diosgenin on oxidative stress in aorta of streptozotocin induced diabetic rats. Eur J Pharmacol 2012; 691: 143-50.

8 Kwon CS, Sohn HY, Kim SH, Kim JH, Son KH, Lee JS, et al. Antiobesity effect of Dioscorea nipponica Makino with lipase-inhibitory activity in rodents. Biosci Biotechnol Biochem 2003; 67: 1451-6.

9 Uemura T, Goto T, Kang MS, Mizoguchi N, Hirai S, Lee JY, et al. Diosgenin, the main aglycon of fenugreek, inhibits LXRalpha activity in HepG2 cells and decreases plasma and hepatic triglycerides in obese diabetic mice. J Nutr 2011; 141: 17-23.

10 Moalic S, Liagre B, Corbiere C, Bianchi A, Dauca M, Bordji K, et al. A plant steroid, diosgenin, induces apoptosis, cell cycle arrest and COX activity in osteosarcoma cells. FEBS Lett 2001; 506: 225-30.

11 Liu MJ, Yue PY, Wang Z, Wong RN. Methyl protodioscin induces $G_{2} / M$ arrest and apoptosis in $\mathrm{K} 562$ cells with the hyperpolarization of mitochondria. Cancer Lett 2005; 224: 229-41.

12 Miyoshi N, Nagasawa T, Mabuchi R, Yasui Y, Wakabayashi K, Tanaka $\mathrm{T}$, et al. Chemoprevention of azoxymethane/dextran sodium sulfateinduced mouse colon carcinogenesis by freeze-dried yam sanyaku 
and its constituent diosgenin. Cancer Prev Res (Phila) 2011; 4: $924-$ 34.

13 Bian D, Li Z, Ma H, Mu S, Ma C, Cui B, et al. Effects of diosgenin on cell proliferation induced by IGF-1 in primary human thyrocytes. Arch Pharm Res 2011; 34: 997-1005.

14 Chen CR, Pichurin P, Nagayama Y, Latrofa F, Rapoport B, McLachlan $\mathrm{SM}$. The thyrotropin receptor autoantigen in Graves disease is the culprit as well as the victim. J Clin Invest 2003; 111: 1897-904.

15 Rajkovic V, Matavulj M, Johansson O. Light and electron microscopic study of the thyroid gland in rats exposed to power-frequency electromagnetic fields. J Exp Biol 2006; 209: 3322-8.

16 Chen CY, Kimura H, Landek-Salgado MA, Hagedorn J, Kimura M, Suzuki K, et al. Regenerative potentials of the murine thyroid in experimental autoimmune thyroiditis: role of CD24. Endocrinology 2009; 150: 492-9.

17 Thoolen B. BrdUrd labeling of S-phase cells in testes and small intestine of mice, using microwave irradiation for immunogold-silver staining: an immunocytochemical study. J Histochem Cytochem 1990; 38: 267-73.

18 Livak KJ, Schmittgen TD. Analysis of relative gene expression data using real-time quantitative PCR and the 2(-Delta Delta $\mathrm{C}(\mathrm{T})$ ) Method. Methods 2001; 25: 402-8.

19 Xavier LL, Viola GG, Ferraz AC, Da Cunha C, Deonizio JM, Netto CA, et al. A simple and fast densitometric method for the analysis of tyrosine hydroxylase immunoreactivity in the substantia nigra pars compacta and in the ventral tegmental area. Brain Res Brain Res Protoc 2005; 16: 58-64.

20 Pyo JS, Kang G, Kim DH, Chae SW, Park C, Kim K, et al. Activation of nuclear factor-kappaB contributes to growth and aggressiveness of papillary thyroid carcinoma. Pathol Res Pract 2013; 209: 228-32.

21 Itoi T, Shinohara Y, Takeda K, Nakamura K, Takei K, Sanada J, et al. Nuclear cyclin D1 overexpression is a critical event associated with cell proliferation and invasive growth in gallbladder carcinogenesis. J Gastroenterol 2000; 35: 142-9.

22 lyengar B. Expression of proliferating cell nuclear antigen (PCNA): proliferative phase functions and malignant transformation of melanocytes. Melanoma Res 1994; 4: 293-5.

23 Nagayama Y, Saitoh O, McLachlan SM, Rapoport B, Kano H, Kumazawa Y. TSH receptor-adenovirus-induced Graves' hyperthyroidism is attenuated in both interferon-gamma and interleukin-4 knockout mice; implications for the Th1/Th2 paradigm. Clin Exp Immunol 2004; 138: 417-22.

24 Misharin A, Hewison M, Chen CR, Lagishetty V, Aliesky HA, Mizutori $\mathrm{Y}$, et al. Vitamin D deficiency modulates Graves' hyperthyroidism induced in BALB/c mice by thyrotropin receptor immunization. Endocrinology 2009; 150: 1051-60.
25 Ueki I, Abiru N, Kobayashi M, Nakahara M, Ichikawa T, Eguchi K, et al. B cell-targeted therapy with anti-CD20 monoclonal antibody in a mouse model of Graves' hyperthyroidism. Clin Exp Immunol 2011; 163: 309-17.

26 Huang $\mathrm{CH}$, Liu DZ, Jan TR. Diosgenin, a plant-derived sapogenin, enhances regulatory T-cell immunity in the intestine of mice with food allergy. J Nat Prod 2010; 73: 1033-7.

27 Huang $\mathrm{CH}$, Ku CY, Jan TR. Diosgenin attenuates allergen-induced intestinal inflammation and IgE production in a murine model of food allergy. Planta Med 2009; 75: 1300-5.

28 Kirik OV, Beznin GV, Korzhevskii DE. Proliferation markers used in histological studies. Morfologiia 2009; 136: 95-100.

29 Volzke H, Friedrich N, Schipf S, Haring R, Ludemann J, Nauck M, et al. Association between serum insulin-like growth factor-l levels and thyroid disorders in a population-based study. J Clin Endocrinol Metab 2007; 92: 4039-45.

30 Maiorano E, Perlino E, Triggiani V, Nacchiero M, Giove E, Ciampolillo A. Insulin-like growth factor-1 and insulin-like growth factor receptor in thyroid tissues of patients with Graves' disease. Int J Mol Med 1998; 2: 483-6.

31 Ren M, Zhong X, Ma CY, Sun Y, Guan QB, Cui B, et al. Insulin-like growth factor-1 promotes cell cycle progression via upregulation of cyclin D1 expression through the phosphatidylinositol 3-kinase/ nuclear factor-kappaB signaling pathway in FRTL thyroid cells. Acta Pharmacol Sin 2009; 30: 113-9.

32 Haddad JJ, Abdel-Karim NE. NF-kappaB cellular and molecular regulatory mechanisms and pathways: therapeutic pattern or pseudoregulation? Cell Immunol 2011; 271: 5-14.

33 Salminen A, Kaarniranta K. Insulin/IGF-1 paradox of aging: regulation via AKT/IKK/NF-kappaB signaling. Cell Signal 2010; 22: 573-7.

$34 \mathrm{Kuo} \mathrm{SH}$, Chou CH, Cheng AL, Wang CW, Chen YH, Chen RJ. Expression of $\mathrm{BCL} 10$ in cervical cancer has a role in the regulation of cell growth through the activation of NF-kappaB-dependent cyclin D1 signaling. Gynecol Oncol 2012; 126: 245-51.

35 Stacey DW. Cyclin D1 serves as a cell cycle regulatory switch in actively proliferating cells. Curr Opin Cell Biol 2003; 15: 158-63.

36 Travali S, Ku DH, Rizzo MG, Ottavio L, Baserga R, Calabretta B. Structure of the human gene for the proliferating cell nuclear antigen. J Biol Chem 1989; 264: 7466-72.

37 Li F, Fernandez PP, Rajendran P, Hui KM, Sethi G. Diosgenin, a steroidal saponin, inhibits STAT3 signaling pathway leading to suppression of proliferation and chemosensitization of human hepatocellular carcinoma cells. Cancer Lett 2010; 292: 197-207.

38 Srinivasan S, Koduru S, Kumar R, Venguswamy G, Kyprianou N, Damodaran C. Diosgenin targets Akt-mediated prosurvival signaling in human breast cancer cells. Int J Cancer 2009; 125: 961-7. 